\title{
VoIP over WiMAX: Quality of Experience Evaluation
}

\author{
Vitor Bernardo, Bruno Sousa, Marilia Curado \\ Laboratory of Communications and Telematics \\ Department of Informatics Engineering, University of Coimbra \\ \{vmbern,bmsousa,marilia\}@dei.uc.pt
}

\begin{abstract}
VoIP applications are being widely used in today's networks challenging their capabilities to provide a good quality of experience level to the users. In particular, new wireless broadband technologies, such as WiMAX, are being deployed and need to be evaluated to check the performance levels of VoIP services. The work presented in this paper is a unique contribution assessing the VoIP sessions quality on a real WiMAX test-bed, using UDP/RTP and DCCP transport protocols. VoIP quality is measured according the quality perceived by the end users as well as through conventional network parameters, such as one-way delay and packet loss. The results put in evidence a good quality for VoIP services with 60 simultaneous users in a WiMAX link with resources pre-provisioned. Moreover, in the scenarios tested, DCCP has not shown enhanced performance when compared with UDP/RTP, despite the congestion control mechanisms natively supported.
\end{abstract}

\section{Introduction}

In the last years, we have witnessed a change of the Internet contents, which include an increasing variety of multimedia applications, with different characteristics. In such a way, the current wireless access technologies address these requirements in their core design.

WiMAX is a broadband wireless technology based on the IEEE 802.16 standards and aims at enabling the development of wireless broadband services anytime and anywhere [1]. WiMAX can be deployed in diverse scenarios such as fixed, portable, nomadic and mobile. Despite that WiMAX is not yet widely deployed, it is a candidate for the last mile access in both urban and rural areas. Thus, it is crucial to assess the capabilities of WiMAX to support multimedia applications, namely, voice over IP (VoIP).

VoIP applications are usually transmitted over the User Datagram Protocol (UDP) associated with the
Real-time Transport Protocol (RTP) [2]. However, the recently developed transport protocol, Datagram Congestion Control Protocol (DCCP), performing congestion control in the network independently of the application, is also a candidate to transmit VoIP traffic [3].

In this context, there are two main aspects that determine the VoIP quality of experience users sharing a WiMAX link, namely, the number of simultaneous clients using the same channel and the respective provisioned bandwidth. The achievement of these challenging objectives includes the study, on a real scenario test-bed, of multiple VoIP sessions, using UDP and DCCP, over the WiMAX technology integrated on an end-to-end IP architecture.

The paper is organized as follows: Section 2 describes the WiMAX technology. The Datagram Congestion Control protocol is introduced in Section 3. Section 4 presents the methodology used to assess the VoIP quality. The results of the evaluation on the testbed are discussed in Section 5, and the relevant related work is presented in Section 6. Finally, Section 7 concludes the paper and presents issues to be addressed in future work.

\section{WiMAX Technology}

WiMAX, Worldwide Interoperability for Microwave Access, is a technology based on the IEEE 802.16 standards and aims at enabling the development of wireless broadband systems. There are two versions of WiMAX, the Fixed WiMAX (based on IEEE 802.16d-2004[4]) and Mobile WiMAX (based on IEEE 802.16e-2005[5]).

The IEEE 802.16 defines the physical (PHY) and medium access control (MAC) layers. The MAC layer plays a crucial role because it is responsible for the most important features of the standard, such as Quality of Service (QoS) implementation.

The IEEE 802.16-2004 standard defines four classes of service: Unsolicited Grant Service (UGS), Real-Time Polling Service (rtPS), Non-Real Time 
Polling Service (nrtPS) and Best Effort (BE). The IEEE 802.16e-2005 extends the classes of service supported by introducing the Extended Real-Time Polling Service (ertPS) class of service.

The WiMAX Forum is the organization responsible for the certification and interoperability of all the products based on the IEEE 802.16 standard family. The Network Working Group of the WiMAX Forum has defined the WiMAX Network Reference Model [1] in order to integrate WiMAX into an all-IP based network, as Figure 1 depicts.

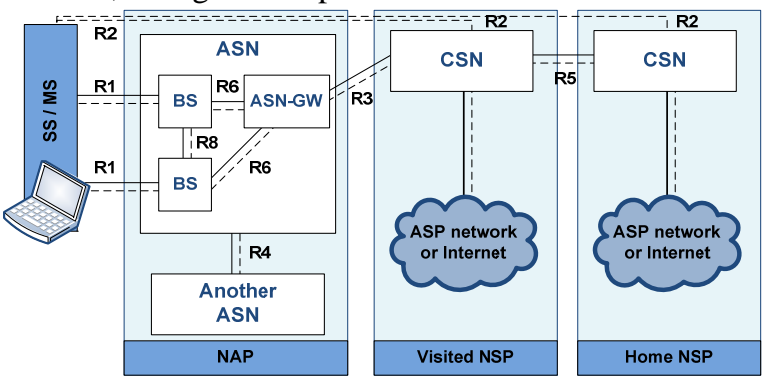

Figure 1 - Network Reference Model [6]

The Network Access Provider (NAP) is the entity that provides WiMAX radio access to the Network Service Provider (NSP) and controls the Access Service Network (ASN). The IP connectivity and the WiMAX services are provided by the Network Service Provider (NSP), which also manages the Connectivity Service Network (CSN). Each Subscriber/Mobile Station (SS/MS) is connected to the ASN through one Base Station (BS).

The communication between the different entities is performed through the reference points R1-R8, specified to provide interoperability. The ASN provides radio access to all the subscribers, while CSN supports the IP connectivity and all the network functions, such as DHCP, AAA among others.

\section{Datagram Congestion Control Protocol}

This section describes the emergent transport protocol Datagram Congestion Control Protocol (DCCP) [3].

Nowadays, the most used transport protocols in Internet are, UDP and the Transport Control Protocol (TCP). Although TCP is reliable, it is not suited for multimedia applications, as the packets received after the playout time are useless. Consequently, such transport is performed by UDP in association with RTP. However, UDP does not have congestion control mechanisms and it is unreliable. Given the above limitations, the Internet Engineering Task Force (IETF) developed a new transport protocol named DCCP. The main goals of this protocol are the establishment, maintenance and teardown of flows. Unlike RTP, which requires the participation of the application in the congestion control process, DCCP aims to be completely application-unaware. This is a key point, because there are many applications that are not prepared to deal with network congestion problems. DCCP supports different congestion control mechanisms, identified by the Congestion Control IDentifier (CCID). Currently, there are two CCIDs: the CCID2 [7] and the CCID3 [8].

\subsection{DCCP CCID2}

This congestion control algorithm is similar to the Additive Increase / Multiplicative Decrease (AIMD) feedback control algorithm used in the TCP Congestion Avoidance. The CCID2 is also known as TCP-like Congestion Control. The basic congestion control is based on the transmission window size adjustment. This profile should be employed when the users want to take advantage of all the available bandwidth within a variable environment.

\subsection{DCCP CCID3}

Unlike CCID2, this is a rate controlled congestion control algorithm, based on the TCP - Friendly Rate Control (TFRC). When compared with TCP, the DCCP CCID3 has very smooth variations on the average throughput. Therefore it is more appropriate for applications that need a smooth sending rate such as voice streaming. The DCCP Working Group is currently developing a new CCID, named CCID4 [9], which is based on the TCP-Friendly Rate Control for Small Packets (TFRC-SP). This experimental profile has a special focus on the performance improvement of the applications which send small packets, such as VoIP.

\section{VoIP quality measurement}

This section presents the minimal requirements at the network level for the VoIP transmission over WiMAX and describes the methodology used to assess the VoIP quality.

\subsection{Minimal network requirements}

The WiMAX Forum has defined a set of minimal requirements according to the applications type, being specified in consonance to the metrics defined by the IETF IP Performance Metrics (IPPM) Working Group [10]. The requirements for VoIP applications are the following:

- Typical Data Rate: 4-384Kbps

- Delay: $<150 \mathrm{~ms}$

- Jitter: $<50 \mathrm{~ms}$

- Packet Loss: $<1 \%$ 
These values represent the minimum threshold and are recommend by the WiMAX Forum to provide an effective good quality of experience to the end-users.

\subsection{E-model}

The most popular mechanism to measure the voice quality is the Mean Opinion Score (MOS) scale. Nevertheless the MOS is a sensorial metric, which requires humans to evaluate the quality of the voice in a scale from 1 (bad quality) to 5 (excellent quality). As an alternative to MOS, the E-Model [11] is a mathematical model based evaluation, which is able to predict the quality of voice from the network parameters, such as delay and packet loss. The EModel's output is called R-Factor. Equation 1 depicts the relationship between MOS and the R-Factor .

$$
\begin{gathered}
\text { MOS }=\left\{\begin{array}{cl}
\alpha \times R(R-60)(100-R) & \\
4.5 & 0<R<100(1)
\end{array}\right. \\
\text { where } \alpha=1+0.035 R+7 \times 10^{-6}
\end{gathered}
$$

The R-Factor can be calculated using the following equation:

$$
\begin{aligned}
& R=R_{o}-I_{s}-I_{d}-I_{e f}-A \\
& \text { with } \\
& \begin{array}{cl}
R_{o} & \text { Signal to noise ratio } \\
I_{s} & \text { Simultaneous impairment factor } \\
I_{d} & \text { Delay impairment factor } \\
I_{e f} & \text { Equipment impairment factor } \\
A & \text { Advantage factor }
\end{array}
\end{aligned}
$$

In [12] the authors have reduced the R-Factor calculation to an equation based only on the delay and loss rate values, as shown in Equation 3.

$$
R=94.2-I_{d}-I_{e f}
$$

Moreover, in [12] the authors have also defined a way to convert the one-way delay $d$ and loss rate $e$ when using G.711 CODEC to $I_{d}$ and $I_{e f}$ as shown, respectively, in Equations 4 and 5.

$$
I_{d}=0.024 d+0.11(d-177.3) \times H(d-177.3)
$$

where $H$ is the unit step function.

$$
I_{e f}=0+30 \times \ln (1+15 e)
$$

The MOS calculations in the following section are founded on the simplified equations here presented.

\section{Experimental Evaluation}

This section presents the main objectives, introduces the test-bed configuration and discusses the obtained results.

\subsection{Objectives}

The main objective of this experimental evaluation is to determine the capabilities of Fixed WiMAX to support VoIP services. Such support is assessed with conventional network performance parameters like one-way delay and packet loss, and, at the same time, the voice quality experienced by the end-users, through the objective calculation of the MOS value.

Moreover, VoIP quality will be assessed in scenarios where transport relies on UDP/RTP and DCCP protocols, in order to compare both approaches.

The number of simultaneous clients using the channel, as well as, the bandwidth reserved to support the respective flows are also tested to evaluate the scalability and the behavior with overestimated and underestimated reservations, respectively.

\subsection{Test-bed Setup}

The configured test-bed is compliant with the Network Reference Model of the WiMAX Forum, as depicted in Figure 2. With this approach, it is possible to evaluate a real-life deployment of WiMAX network solution.

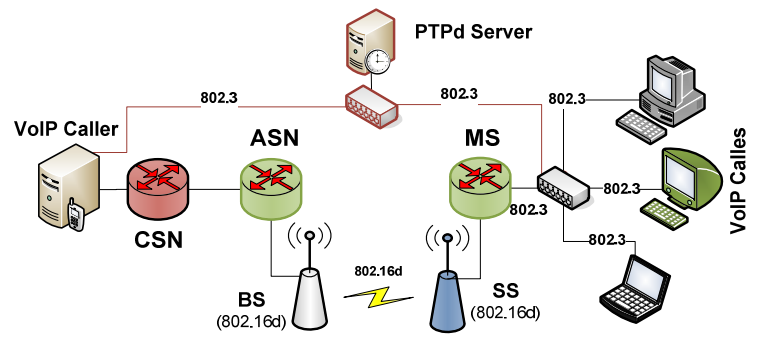

Figure 2 - Fixed WiMAX test-bed

The WiMAX equipment consists on a RedLine Communications AN100U Base Station (BS-1) and a RedLine Communications Subscriber Unit - Outdoor (SS-1). The non WiMAX entities are machines running Linux with kernel 2.6.27-7. The VoIP calls are simulated by the Distributed Internet Traffic Generator (D-ITG) [13]. D-ITG was chosen as it supports DCCP when compared to other available traffic generators. In addition, the Precision Time Protocol daemon (PTPd) [14] was employed for clock synchronization.

\subsection{Tests Description}

The tests were performed using a machine in the CSN network, which acts as a caller and three machines connected to the SS through the MS. These later machines act as the callees of the VoIP calls. To 
assess the performance of VoIP, D-ITG was configured with the G.711 CODEC with a compression rate of $64000 \mathrm{bps}$ and a sample interval of $10 \mathrm{~ms}$ and with one sample per packet. Each test has 60 seconds of duration.

As stated before, one of the key features of WiMAX is the native QoS support, on which different QoS classes are intended for different kind of applications. The WiMAX RedLine equipment used in our test-bed only supports Best Effort and rtPS scheduling classes. Therefore, VoIP was evaluated using the rtPS service, since the Best Effort class does not provide any QoS assurance.

A set of preliminary tests were performed in the Base Station in order to establish the minimal bandwidth required for each client set.

When the bandwidth reservation is overestimated, the flows in the uplink and downlink directions have $9000 \mathrm{kbps}$ allocated. When underestimated the downlink reservation is configured according to the values presented in the Table 1 while the uplink service flow is constant (9000kbps). Moreover, the maximum latency configured for each flow is $300 \mathrm{~ms}$.

Table 1 - Reservations performed in underestimated tests

\begin{tabular}{|c|c|}
\hline $\begin{array}{c}\text { Number of } \\
\text { Flows }\end{array}$ & $\begin{array}{c}\text { Downlink Reservation } \\
\text { (kbps) }\end{array}$ \\
\hline 1 & 100 \\
\hline 10 & 1000 \\
\hline 20 & 2000 \\
\hline 30 & 3000 \\
\hline 40 & 4000 \\
\hline 50 & 5000 \\
\hline 60 & 6000 \\
\hline 70 & 7000 \\
\hline
\end{tabular}

The DCCP configuration parameters (e.g. seq_window) are based on the default of the Linux kernel for both CCID2 and CCID3 algorithms. All the values presented for each test in the following sections are determined on the average of ten multiple runs.

\subsection{Results}

This section presents the results for both overestimated and underestimated scenarios.

\subsubsection{Overestimated Bandwidth Scenario}

This section aims to evaluate the capability of Fixed WiMAX to transmit VoIP flows under very good conditions, i.e., when the bandwidth allocated is higher than the required.

Figure 3 depicts the one-way delay of the VoIP calls for the different clients set and for each transport protocol tested. The line outlined in the $150 \mathrm{~ms}$ represents the maximum acceptable one-way delay, as recommended by ITU in G.114 [15].

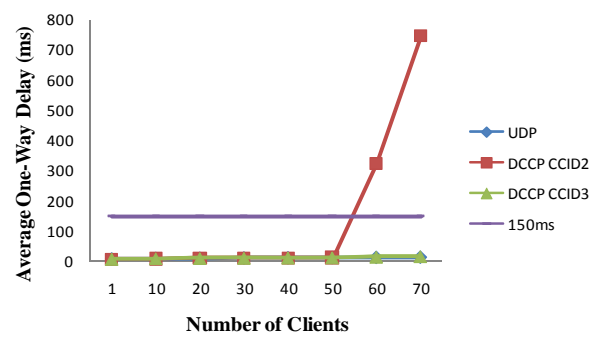

Figure 3 - One-way delay (Overestimated)

The average one-way delay increases slightly for both UDP and DCCP with CCID3 as the number of clients increments. However, when using DCCP with CCID2, the one-way average delay for 60 and 70 clients increases quickly, exceeding the reference value of $150 \mathrm{~ms}$. Such fact is due to the TCP Like mechanisms, which require more bandwidth in the uplink channel. This behavior is explained as follows: the base station uses Time Division Duplexing (TDD) to transmit and receive in the same radio frequency channel. All the up and downlink transmissions are scheduled by the base station. Thus, the uplink has some impact on the downlink transmission, because both transmissions share the same radio frequency channel. When the number of clients increases the number of control messages also increases. Consequently, the WiMAX Base Station must schedule the down and uplink transmission in the time and this causes a larger delay in the transmission.

As presented in Figure 4, packet loss is inexistent or is below the reference value of $1 \%$ in almost all the cases, with the exception of DCCP CCID2 with more than 50 clients. This behavior is due to the congestion control mechanism, which is based on the adjustment of the window size, and has shown poor results in these situations.

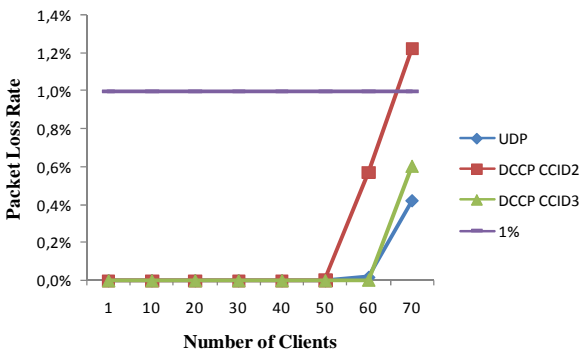

Figure 4 - Packet Loss Rate (Overestimated)

Figure 5 pictures the relationship between the number of clients and the Mean Opinion Score. The voice quality is very good for up to 60 clients with UDP and DCCP CCID3. However, UDP is able to provide a slightly better quality than DCCP CCID3, even when the voice quality becomes poor (i.e. with 70 clients). 


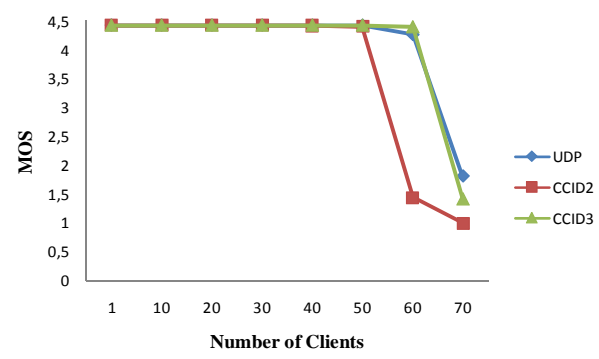

Figure 5 - MOS (Overestimated)

The results have shown that Fixed WiMAX is able to provide a very good quality of voice, when the number of simultaneous clients is lower than 70 . However, even with an overestimated reservation channel of 9Mbps and using the UDP protocol, which uses only 7280Kbps of the downlink and 0Kbps of the uplink, the voice quality with 70 simultaneous clients is poor.

\subsubsection{Underestimated Bandwidth Scenario}

In this scenario the reserved bandwidth is always lower than the required by the different client sets.

Figure 6 and Figure 7 show the average delay and the packet loss rate, respectively, for the different number of clients.

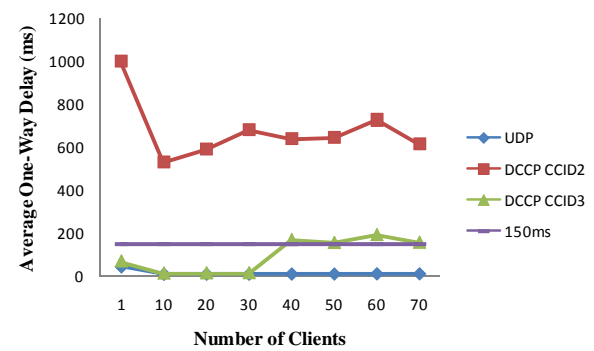

Figure 6 - One-way delay (Underestimated)

Flows with UDP have always one-way delay below 150ms. The DCCP CCID3 has a similar behavior for up to 30 clients. As the number of clients increases, DCCP CCID3 has a smaller packet loss rate, at the expense of the one-way delay, which increases proportionally. DCCP CCID2 has always poor performance.

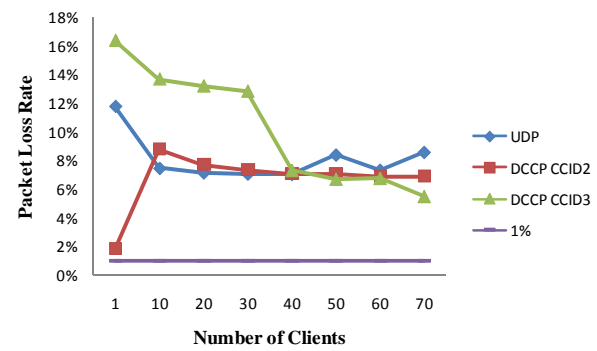

Figure 7 - Packet Loss Rate (Underestimated)
When DCCP is used, there is a relationship between the one-way delay and the packet loss. If the protocol responds rapidly to the congestion in the network, it will reduce the transfer rate in order to minimize the packet loss. However, as seen in Figure 7, to reduce the packet loss with 1 client, the DCCP CCID2 increases the one-way delay on the transmission. This is not feasible when transmitting VoIP or other kind of real time application traffic. None of the tested protocols is able to grant a packet loss below $1 \%$, which is the recommendation of the WiMAX Forum for the transmission of VoIP in good conditions.

The quality measured by the calculation of the RFactor shows an R-Factor always lower than zero. So, the MOS value is always equal to one, which means that the voice quality perceived by the clients was very bad.

\section{Related work}

This section presents the related work inline with the evaluation performed in this paper.

Balan, H.V. et al, perform an experimental evaluation of voice quality over DCCP in [16]. The experimental setup compares UDP, TCP, DCCP CCID3 and TFRC. The main conclusions are that any of the proposals of TFRC can achieve the same voice quality as UDP.

An experimental study of DCCP for multimedia applications, with focus on video, is shown in [17]. The general conclusions of both works are that DCCP deteriorated in some way the transmission quality.

Melo de Sales et al. have performed a test-bed evaluation of TCP, UDP and DCCP over 802.11g networks on a real test-bed [18]. The main conclusions are that DCCP decreases throughput and delivers some out-of-order packets. However, the tests were not performed with data-oriented applications.

The VoIP quality on a WiMAX test-bed is discussed in [19]. This study is limited to the transmission over UDP and the maximum number of concurrent flows presented for each subscriber station is only 20, which is rather limited to evaluate simultaneously the scalability and the quality. The main conclusions are that WiMAX is able to fit quiet well the needs of VoIP transmissions.

After the analysis of the related work and to the best of our knowledge, the work presented in this paper is a unique contribution assessing the quality of experience of VoIP sessions, using both UDP/RTP and DCCP transport protocols, on a real WiMAX test-bed.

\section{Conclusion and Further work}

As multimedia applications are becoming popular and wireless broadband technologies are deployed, 
there is the need to assess the quality of experience experienced by users. This paper has presented an evaluation of VoIP sessions on a fixed WiMAX testbed, comparing the performance of UDP/RTP and DCCP in overestimated and underestimated scenarios.

The overestimated bandwidth scenario showed that Fixed WiMAX is able to provide very good quality for VoIP services. The support of multiple simultaneous clients is good in almost all the cases when adequate resources were allocated. However, when resources were underestimated, all the clients have a poor voice quality due to the network congestion problems, as reported by the measured values of one-way delay and packet loss.

DCCP CCID2 has worst performance than UDP and DCCP CCID3, when the number of clients is high. Moreover, both DCCP control congestion algorithms use more bandwidth than UDP and do not provide any additional performance benefit. The comparison of the protocols in the underestimated scenario showed that, even without being enough to ensure the quality of VoIP applications, UDP has better performance. In short, in these scenarios UDP has shown a better cost/benefit ratio.

In both scenarios it is possible to establish a relationship between the evaluated network parameters such as one-way delay, jitter and packet loss rate and the MOS value. And, as expected, when the network parameters are below the recommendations of WiMAX Forum the quality of voice perceived by the end-users is poor.

In the future, DCCP tests with CCID4 will be performed, in order to evaluate its capability of transport VoIP on WiMAX. In the performed tests DCCP has not shown any advantage over UDP and has introduced a significant overhead. However, DCCP has the potential to achieve better results in a scenario with alternate overestimated and underestimated bandwidth reservations, i.e. in dynamic situations. This scenario will also be evaluated in future work.

\section{References}

[1] WiMAX Forum Network Architecture (Stage 2: Architecture Tenets, Reference Model and Reference Poins). Release 1, version 1.2. WiMAX Forum, January 11, 2008

[2] H. Schulzrinne, S. Casner, R. Frederick, V. Jacobson, "RTP: A Transport Protocol for Real-Time Applications”, RFC 3550, IETF Network Working Group, July 2003

[3] E. Kohler,M. Handley, S. Floyd, "Datagram Congestion Control Protocol (DCCP)”, RFC 4340, IETF Network Working Group, March 2006
[4] "IEEE Standard for Local and Metropolitan Area Networks Part 16: Air Interface for Fixed Broadband Wireless Access Systems," IEEE Std 802.16-2004 (Revision of IEEE Std 802.16-2001) , vol., no., pp. 0_1857, 2004

[5] "IEEE Standard for Local and Metropolitan Area Networks Part 16: Air Interface to fixed and Mobile Broadband Wireless Access Systems", IEEE Std 802.16-2005, 2005

[6] K. Etemad, "Overview of Mobile WiMAX technology and evolution", IEEE Communications Magazine, October 2008

[7] S. Floyd, E. Kohler, "Profile for Datagram Congestion Control Protocol (DCCP) Congestion Control ID 2: TCP-like Congestion Control” RFC 4341 (Proposed Standard). IETF Network Working Group, March 2006

[8] S. Floyd, E. Kohler, J. Padhye, "Profile for Datagram Congestion Control Protocol (DCCP) Congestion Control ID 3: TCP-Friendly Rate Control (TFRC)” RFC 4342 (Proposed Standard). Updated by RFC 5348. September 2008

[9] S. Floyd, E. Kohler, "Profile for Datagram Congestion Control Protocol (DCCP) Congestion ID 4: TCPFriendly Rate Control for Small Packets (TFRC-SP)". draft-ietfdccp-ccid4-02.txt, 9 February 2008

[10] WiMAX Forum, "WiMAX System Evaluation Methodology”, 2008

[11] ITU-T Recommendation G.107, "The E-model, a computational model for use in transmission planning", 2005

[12] R.G. Cole, J. H. Rosenbluth, "Voice over IP performance monitoring”. SIGCOMM Comput. Commun. Rev., 31(2), 9-24, 2001

[13] A. Botta, A. Dainotti, A. Pescapè, "Multi-protocol and multi-platform traffic generation and measurement", INFOCOM 2007 DEMO Session, May 2007, Anchorage, Alaska, USA

[14] K. Correll, N. Barendt, "PTPd - Precision Time Protocol daemon”. http://ptpd.sourceforge.net/. Last visit: 16 January 2009.

[15] ITU-T, ITU-T Recommendation G.114, One-Way transmission time, 2003

[16] H.V Balan, L. Eggert, S. Niccolini, M. Brunner, M., "An experimental evaluation of voice quality over the Datagram Congestion Control Protocol”, Proc. IEEE INFOCOM 6-12, 2007

[17] F. Nivor, "Experimental study of DCCP for multimedia applications", ACM conference on Emerging network experiment and technology, ACM New York, NY, USA, 2005

[18] L.M. de Sales, H.O. Almeida, A. Perkusich, "On the performance of TCP, UDP and DCCP over $802.11 \mathrm{~g}$ networks”, Proceedings of the 2008 ACM symposium on Applied computing, 2008

[19] N. Scalabrino, F. De Pellegrini, R. Riggio, A. Maestrini, C. Costa, I. Chlamtac, "Measuring the quality of VoIP traffic on a WiMAX testbed", IEE TridentCom 2007, May 2007 